\title{
Pronóstico de quiebra y valuación de acciones en empresas sustentables de México en la era COVID19
}

DOI: https://doi.org/10.17981/econcuc.42.2.2021.Econ.1

Artículo de investigación científica y tecnológica. Fecha de recepción: 31/07/2020

Fecha de devolución: 22/11/2020

Fecha de aceptación: 03/12/2020

Fecha de publicación: 01/03/2021

Arturo Morales Castro

Universidad Nacional Autónoma de México

Ciudad de México, Estado de México (México) amorales@fca.unvam.mx

\section{Sandra Avendaño Cruz}

Universidad Nacional Autónoma de México Ciudad de México, Estado de México (México) sandyuamyunam@gmail.com

\section{José Miguel Mata Hernández}

Universidad Nacional Autónoma de México

Ciudad de México, Estado de México (México) josemiguelmata@hotmail.com

\section{Resumen}

En los años recientes las empresas sustentables han sido un gran auge en cuanto a inversión y medidas sostenibles por las mismas, es por ello por lo que varios inversionistas han buscado conformar sus portafolios de inversión con empresas sustentables. Por sustentable se entiende como el proceso que busca lograr una prosperidad económica y el equilibrio entre el medio ambiente y el uso de los recursos naturales. El S\&P/BMV Total México ESG Índex de la Bolsa Mexicana de Valores agrupa a varias empresas que poseen las mejores prácticas en desempeño ambiental, social y de gobierno corporativo, esto con el objetivo de formar una economía sólida, que sea adecuada al contexto actual y las necesidades del futuro. El estudio de la presente investigación se enfoca al análisis de empresas que pertenecen al S\&P/BMV Total México ESG Índex de la Bolsa Mexicana de Valores, con el objetivo de aplicar el cálculo de Q de Tobin que fue propuesta por Tobin (1969), la cual consiste en determinar si la acción de la empresas están subvaluadas, sobrevaluadas o son valoradas correctamente y por otro lado el cálculo de Z score de Altman que fue propuesta por Altman (1968), el cual consiste en pronosticar la quiebra en las empresas, estos cálculos se utilizan para observar si las empresas que pertenecen a este índice tienen: salud financiera, si el valor de la acción de estas entidades está sobrevalorado o infravalorado y las probabilidades de quebrar que tengan estas mismas firmas. Se concluye que a raíz del COVID-19 los Resultados Financieros del primer trimestre de 2020 se vieron afectados y por tanto las empresas están en riesgo de quiebra y otras por su posicionamiento y expansión no se vieron afectadas de acuerdo con los parámetros de los modelos mencionados.

Palabras clave: Inversión; mercado de valores; sustentabilidad; quiebra; valuación de acciones; valoración de inversiones; Z score; Q de Tobin

\begin{abstract}
In recent years, sustainable companies have been a great boom in terms of investment and sustainable measures by them, which is why several investors have sought to shape their investment portfolios with sustainable companies. Sustainable is understood as the process that seeks to achieve economic prosperity and the balance between the environment and the use of natural resources. The S\&P/BMV Total Mexico ESG Index of the Mexican Stock Exchange brings together several companies that have the best practices in environmental, social and corporate governance performance, this with the aim of forming a solid economy that is appropriate to the current context and the needs of the future. The study of this research focuses on the analysis of companies that belong to the S\&P/BMV Total Mexico ESG Index of the Mexican Stock Exchange, with the aim of applying the Tobin Q calculation that was proposed by Tobin (1969), the which consists of determining if the shares of the companies are undervalued, overvalued or are valued correctly and, on the other hand, the calculation of Altman's Z score that was proposed by Altman (1968), which consists of forecasting bankruptcy in companies, These calculations are used to observe if the companies that belong to this index have: financial health, if the share value of these entities is overvalued or undervalued and the probabilities of bankruptcy that these same firms have. It is concluded that as a result of COVID-19 the Financial Statements were affected and therefore the companies analyzed are at risk of bankruptcy according to the parameters of the aforementioned models Stock market, Sustainable, Investment.

Keywords: Investment; Stock market; sustainability; bankruptcy; stock valuation; investment valuation; Z score; Tobin's Q
\end{abstract}




\section{INTRODUCCIÓN}

La inversión socialmente responsable es aquella inversión que no solo considera cuestiones de rentabilidad, sino que también considera el impacto social y del medio ambiente. La sustentabilidad implementada por una empresa consiste en la administración eficiente y de forma racional de los recursos, estos ya sean naturales, económicos y sociales, esto con el fin de mejorar el bienestar de la población presente y mejorar la calidad de vida de próximas generaciones. Para una entidad se busca la eficiencia de los recursos, del capital, del mejorar su desarrollo y mejoramiento con la relación social y de sus clientes como de posibles inversionistas.

Las empresas socialmente responsables son aquellas organizaciones que comprenden el valor del bien común como del bien social y no conciben una mejor forma de su desarrollo que no sea de manera sustentable, esto de acuerdo con Chirinos, Fernández \& Sánchez (2012), Pérez, Chumaceiro y Acosta (2019) y Paz, Sierra y López 2019).

La globalización y el auge de empresas con criterios ESG en el país ha llevado a la Bolsa Mexicana de Valores (BMV, 2020) a revisar y a crear índices y productos que cumplan con las normas sostenibles internacionales, esto para adaptarse a recomendaciones emitidas y a nuevos cambios de acuerdo con los organismos internacionales y para mayor captación de clientes. Esto comentado, fue motivo para la creación del S\&P/BMV Total Mexico ESG Index, este índice agrupa a entidades que cumplen con criterios ESG, que en sus siglas en inglés significa Environmental, social and governance, o conocidas en español como los criterios ISR por sus siglas que significan Inversiones Socialmente Responsables. Estas entidades que conforman este índice no incluyen aquellas que se dedican a las industrias de armas convencionales como de tabaco, así también las que no cumplen con los requisitos del Pacto Mundial de Naciones Unidas.

La Bolsa Mexicana de Valores, analizando las tendencias internacionales y el crecimiento que se ha presentado en los mercados financieros especialmente los accionarios en el tema ambiental, social, decidió incorporar el S\&P/BMV Total México ESG Index, a partir de junio del 2020, esto para seguir el comportamiento bursátil, de las emisoras que tienen criterios ESG en el país. Los criterios de selección utilizados para las entidades que conforman este índice son basados en principios ESG pertinentes, este índice de referencia este compuesto por acciones y FIBRAS listadas en la Bolsa Mexicana de Valores.

Las inversiones en emisoras que cotizan en la bolsa mexicana de valores siempre han existido, pero el problema se hace notorio cuando un inversionista quisiera invertir en alguna de las emisoras que forma parte del S\&P/BMV Total Mexico ESG Index, por lo cual, con el cálculo, la interpretación, el análisis y validación de la razón de la $Q$ de Tobin y de la $Z$ score de Altman se pueden dar más herramientas al inversor para una seguridad mayor. Producto de lo comentado anteriormente surge la pregunta de investigación siguiente: ¿El COVID-19 es elemento relevante que afecta la valuación de empresas del IPC Sustentable, haciendo análisis a través de $\mathrm{Q}$ de Tobin y Z de Altman? 
La inversión de capital y de compra de acciones de empresas que entran como entidades sustentables ha crecido de forma exponencial en los últimos años. Dentro de las entidades que conforman el S\&P/BMV Total Mexico ESG Index se encuentran transnacionales, las cuales el tener la insignia y ser consideradas como empresas socialmente responsables, les ha beneficiado en cuanto a su imagen pública, así como la forma en que las ven los clientes, lo cual causa una buena postura y también llega a provocar aumento en ventas.

La investigación amerita el demostrar si una entidad que pertenece al S\&P/BMV Total Mexico ESG Index el precio de su acción se desarrollada de forma óptima, donde su mismo precio demuestra buenas oportunidades de inversión, así como demostrar si el precio de acción de una entidad se encuentra sobrevalorada o infravalorada.

Se analiza también si las firmas que pertenecen a este índice están en riesgo de quiebra (Tabla 1).

TABLA 1.

Objetivos e hipótesis de la investigación.

Objetivos generales

Hipótesis

Evaluar si las empresas que conforman el índice sustentable son sostenibles y hay oportunidad de inversión en ellas de acuerdo con la razón de $\mathrm{Q}$ de Tobin y Z score de Altman. Demostrar si el COVID-19 afectó a las empresas, determinándolo a través del análisis de la $\mathrm{Q}$ de Tobin y la $\mathrm{Z}$ de Altman.

Objetivos específicos.

Observar el comportamiento del precio de las acciones de la muestra de las entidades del índice sustentable antes (2019 4T)

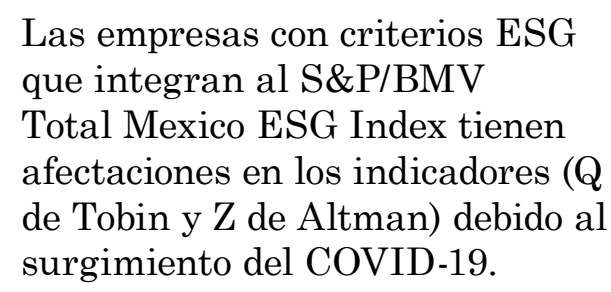
que integran al S\&P/BMV

Total Mexico ESG Index tienen afectaciones en los indicadores $(\mathrm{Q}$ de Tobin y Z de Altman) debido al surgimiento del COVID-19. y después (2020 1T).

Nota. Elaboración propia.

\section{METODOLOGíA}

Para el desarrollo de la investigación los Estados Financieros y las cotizaciones de las empresas se tomaron de Economatica (2020), en miles de pesos y con una periodicidad trimestral del 2019 4T y 2020 1T, para llevar a cabo los cálculos se utilizó Excel y los modelos de $Q$ de Tobin y de la $Z$ de Altman. Se seleccionaron 21 empresas de un total de 29 empresas listadas en el IPC'S, ya que se descartaron 8 pertenecientes al sector financiero (BSMX B, CREAL, FUNO 11, GENTERA, GFNORTE O, FIBRAPL 14, Q y RA).

\section{$Q$ de Tobin}

La razón de es la expresión matemática que muestra la relación entre el valor de mercado de la entidad divido por el valor contable de los activos de la misma entidad. La $Q$ de tobin acuerdo con Langebaek y Ortiz (2007) indican si el activo de la 
entidad está sobrevalorado o infravalorado y esta puede servir para predecir si la inversión de capital aumentará o disminuirá.

Milei (2011) de acuerdo con los estudios de James Tobin comenta que, si la Razón de $Q$ es mayor que 1, significa que la acción está sobrevalorada, por lo tanto, indica que el precio de la acción en el mercado es mayor que el valor patrimonial de la entidad. En el caso contrario en que la Razón de $Q$ sea menor que 1, indica que la acción de la entidad está infravalorada, por lo tanto, el valor en el mercado de esta es menor que el valor patrimonial.

La fórmula de la es la siguiente (1):

$$
\text { Razón } Q \text { de tobin }=\frac{\text { valor de la acción }}{\text { valor contable de la acción }}
$$

Los valores que se deben de tomar en cuenta de acuerdo con los resultados obtenidos son los siguientes:

Razón $Q$ de tobin $>1$ = sobrevalorada.

Razón $Q$ de tobin $<1$ = infravalorada.

Un valor de la razón $Q$ inferior a uno predice que es óptimo para la empresa no invertir más y eliminar capacidad productiva.

\section{$Z$ de Altman}

La $Z$ de Altman mide las probabilidades de que quiebre una empresa de acuerdo con ciertas razones financieras que son razones de liquidez, razones de apalancamiento, razones de actividad y razones de rentabilidad. Anjum (2012) comenta que se puede decir con seguridad que el Modelo $Z$ de Altman se puede aplicar a la economía moderna para predecir la angustia y la quiebra de las empresas con uno, dos o hasta tres años de anticipación.

La fórmula de $Z$ score de Altman para esta investigación consiste en el siguiente cálculo (2):

$$
Z \text { score de Altman }=(A+B+C+D+E)
$$

Donde:

$A=$ (capital neto de trabajo/total de activos) $* 1.2$

$B=$ (utilidades acumuladas /total de activos) $* 1.4$

$C=($ utilidad antes de impuestos e intereses/total de activos $) * 3.3$

$D=($ Valor de mercado / pasivos totales $) * 0.6$

$E=$ (ventas / total de activos) $* 1.0$

De acuerdo con el modelo de , la probabilidad de quiebra de una empresa dependerá del resultado obtenido. De acuerdo con el resultado obtenido, la entidad podría encontrarse en una zona de no riesgo, una zona de alerta o en una zona de peligro. 
- Resultado superior a 2.99 daría como zona de no riesgo

- Resultado entre 1.81 y 2.99 daría como zona de alerta, hay posibilidad que la empresa pueda quebrar en los próximamente o en 2 a 3 años.

- Resultado inferior a 1.81 daría como zona de peligro de una posible quiebra inminente.

\section{DesarRollo y AnÁlisis}

La razón $Q$ de Tobin nos informa sobre las oportunidades de beneficio de una empresa, por lo tanto, sobre sus posibilidades de supervivencia como empresa rentable. Demuestra de esta manera cual es la relación que existe entre su precio en el mercado.

El análisis de ambos indicadores se muestra antes del COVID-19 (2019 4T) y durante el COVID 19 (2020 1T), de esta manera observamos el comportamiento que tiene esta enfermedad en los estados financieros de las empresas seleccionadas (Tabla 2).

TABLA 2

$Q$ de Tobin y $Z$ Score para $20194 T$

\begin{tabular}{|c|c|c|c|}
\hline Empresa & Año & $\mathrm{Q}$ de Tobin & Z Score \\
\hline Alfa A & $20194 \mathrm{~T}$ & 0.5660 & 1.7155 \\
\hline Arca Continental * & $20194 \mathrm{~T}$ & 2.3911 & 2.4861 \\
\hline Rotoplas * & $20194 \mathrm{~T}$ & 2.8943 & 2.4501 \\
\hline Alsea * & $20194 \mathrm{~T}$ & 2.5033 & 1.2044 \\
\hline Asureste B & $20194 \mathrm{~T}$ & 208.4898 & 4.5320 \\
\hline Bimbo A & $20194 \mathrm{~T}$ & 1.8614 & 2.0643 \\
\hline Cemex CPO & $20194 \mathrm{~T}$ & 1.6099 & 1.1518 \\
\hline Fomento Econ Mex UBD & $20194 \mathrm{~T}$ & 27.9148 & 7.7557 \\
\hline Gpo Aeroport Pacif B & $20194 \mathrm{~T}$ & 8.8696 & 4.8875 \\
\hline Herdez * & $20194 \mathrm{~T}$ & 2.6603 & 2.0902 \\
\hline Infraes Energetica * & $20194 \mathrm{~T}$ & 1.5546 & 1.3019 \\
\hline Kimberly Clark Mex A & $20194 \mathrm{~T}$ & 6.6923 & 3.9197 \\
\hline Coca Cola Femsa UBL & $20194 \mathrm{~T}$ & 22.2329 & 7.8753 \\
\hline Genomma Lab Intern B & $20194 \mathrm{~T}$ & 9.0801 & 2.8688 \\
\hline Tenedora Nemak A & $20194 \mathrm{~T}$ & 0.4961 & 1.4982 \\
\hline OMA B & $20194 \mathrm{~T}$ & 20.9924 & 6.4478 \\
\hline Orbia * & $20194 \mathrm{~T}$ & 1.2904 & 1.5269 \\
\hline Penoles Industrias * & $20194 \mathrm{~T}$ & 0.8389 & 2.1479 \\
\hline Televisa Gpo CPO & $20194 \mathrm{~T}$ & 179.5712 & 49.2466 \\
\hline Vesta * & $20194 \mathrm{~T}$ & 332.7335 & 1.1400 \\
\hline Wal Mart de México * & $20194 \mathrm{~T}$ & 7.1215 & 5.9049 \\
\hline
\end{tabular}

Fuente: Elaboración propia a partir de datos obtenidos en Economatica (2020). 
Con resultados de 2019 4T, observamos que, para la razón Q de Tobin, tres empresas tienen una $Q$ menor a 1: Alfa A, Tenedora Nemak A y Peñoles Industrias*, lo que predice que es óptimo para la empresa desinvertir y eliminar capacidad productiva, para seguir operando. Esto como resultado para Alfa A se debe a que en $20294 \mathrm{~T}$ tuvo 339708582 en activo total y para $20201 \mathrm{~T} 426811820$, esto junto con su deuda financiera a corto plazo de 2537867 en 2019 4T a 23269603 para 2020 1T. Para Tenedora Nemak A tiene un resultado menor a 1 ya que su deuda financiera a corto plazo aumento de manera considerable, en el cuarto trimestre 2019 fue de 1976653 y para el primer trimestre $2020 \mathrm{su}$ deuda fue de 10659280 . Y por último para Peñoles su deuda a corto plazo para 20194 T fue de 171,589 y para el primer trimestre 2020 fue de 3385648 (Economatica, 2020).

Analizando la Z de Altman siete empresas se encuentran en $\mathrm{Z}<1.81$ : Alfa A, Alsea*, Cemex CPO, Infraes Energetica*, Tenedora Nemak A, Orbia* y Vesta*, lo que indica que estas están en peligro de quiebra o probabilidad de caer en insolvencia. Con un valor $\mathrm{Z}>2.99$ tenemos a siete empresas: Asureste B, Gpo Aeroport Pacif B, Kimberly Clark Mex A, Coca Cola Femsa UBL, OMA B, Televisa Gpo CPO y Wal Mart de México*, estas empresas son sanas o se ubican en zona de no peligro (Tabla 3).

TABLA 3.

$Q$ de Tobin y Z Score para $20201 T$.

\begin{tabular}{|c|c|c|c|}
\hline Empresa & Año & $\mathrm{Q}$ de Tobin & Z Score \\
\hline Alfa A & $20201 \mathrm{~T}$ & 0.2032 & 0.8794 \\
\hline Arca Continental * & $20201 \mathrm{~T}$ & 1.9684 & 1.6137 \\
\hline Rotoplas * & $20201 \mathrm{~T}$ & 2.5285 & 1.7655 \\
\hline Alsea * & $20201 \mathrm{~T}$ & 0.6952 & 0.4568 \\
\hline Asureste B & $20201 \mathrm{~T}$ & 122.7125 & 2.7384 \\
\hline Bimbo A & $20201 \mathrm{~T}$ & 1.6327 & 1.1084 \\
\hline Cemex CPO & $20201 \mathrm{~T}$ & 0.9422 & 0.7169 \\
\hline Fomento Econ Mex UBD & $20201 \mathrm{~T}$ & 21.7268 & 4.7346 \\
\hline Gpo Aeroport Pacif B & $20201 \mathrm{~T}$ & 4.7247 & 2.8165 \\
\hline Herdez ${ }^{*}$ & $20201 \mathrm{~T}$ & 2.2509 & 0.9375 \\
\hline Infraes Energetica* & $20201 \mathrm{~T}$ & 1.0008 & 0.9576 \\
\hline Kimberly Clark Mex A & $20201 \mathrm{~T}$ & 6.5815 & 2.4152 \\
\hline Coca Cola Femsa UBL & $20201 \mathrm{~T}$ & 17.9447 & 5.3006 \\
\hline Genomma Lab Intern B & $20201 \mathrm{~T}$ & 8.6998 & 2.3826 \\
\hline Tenedora Nemak A & $20201 \mathrm{~T}$ & 0.2068 & 0.8087 \\
\hline OMA B & $20201 \mathrm{~T}$ & 11.9056 & 3.8668 \\
\hline Orbia * & $20201 \mathrm{~T}$ & 0.7082 & 0.9397 \\
\hline Penoles Industrias * & $20201 \mathrm{~T}$ & 0.5473 & 1.0131 \\
\hline Televisa Gpo CPO & $20201 \mathrm{~T}$ & 108.1329 & 24.5012 \\
\hline Vesta * & $20201 \mathrm{~T}$ & 245.3537 & 0.6920 \\
\hline Wal Mart de México* & $20201 \mathrm{~T}$ & 6.9812 & 4.1186 \\
\hline
\end{tabular}

Fuente: Elaboración propia a partir de datos obtenidos de Economatica (2020). 
Con resultados de 2020 1T, se obtuvo que, para la Q de Tobin, seis empresas tienen una Q menor a 1: Alfa A, Alsea*, Cemex CPO, Tenedora Nemak A, Orbia* y Penoles Industrias*, lo que muestra que en presencia del COVID-19 se afectaron a las empresas mencionadas.

Con el indicador $\mathrm{Z}<1.81$, en presencia del COVID-19 aumenta el número de empresas en quiebra inminente o por caer en insolvencia, de siete en 2019 4T, tenemos 12 empresas afectadas para el 2020 1T: Alfa A, Arca Continental", Rotoplas*, Alsea*, Bimbo A, Cemex CPO, Herdez*, Infraes Energetica*, Tenedora Nemak A, Orbia*, Penoles Industrias* y Vesta*. Es decir, en presencia de la Contingencia Sanitaria se ven afectados los Estados Financieros de las empresas analizadas.

Las empresas que se ubican en zona segura durante la contingencia sanitaria (COVID-19) son solo cinco: Fomento Econ Mex UBD, Coca Cola Femsa UBL, OMA B, Televisa Gpo CPO y Wal Mart de México*, pues su valor de Z en presencia de disminuciones se encuentra por arriba de 2.99, lo que indica que son empresas sanas, esto como resultado de su posicionamiento y su nivel de inversión, ventas y activos.

En la Figura 1 se busca comparar las empresas seleccionadas que cotizan en el mercado, con este se visualiza el comportamiento y así se determinan las expectativas sobre beneficios futuros que el mercado inversor posee para las empresas en cuestión, al calcular la q de las empresas, los inversionistas observan las oportunidades que el mercado y la competencia ofrecen a esa empresa con que cuenta internamente para explotarlas. El análisis del comportamiento del IPC'S de México permite conocer el entorno en el que se desenvuelve la Q de Tobin antes y durante el COVID-19.

\section{Comparativo del Valor Q de Tobin 20194T y 20201T}

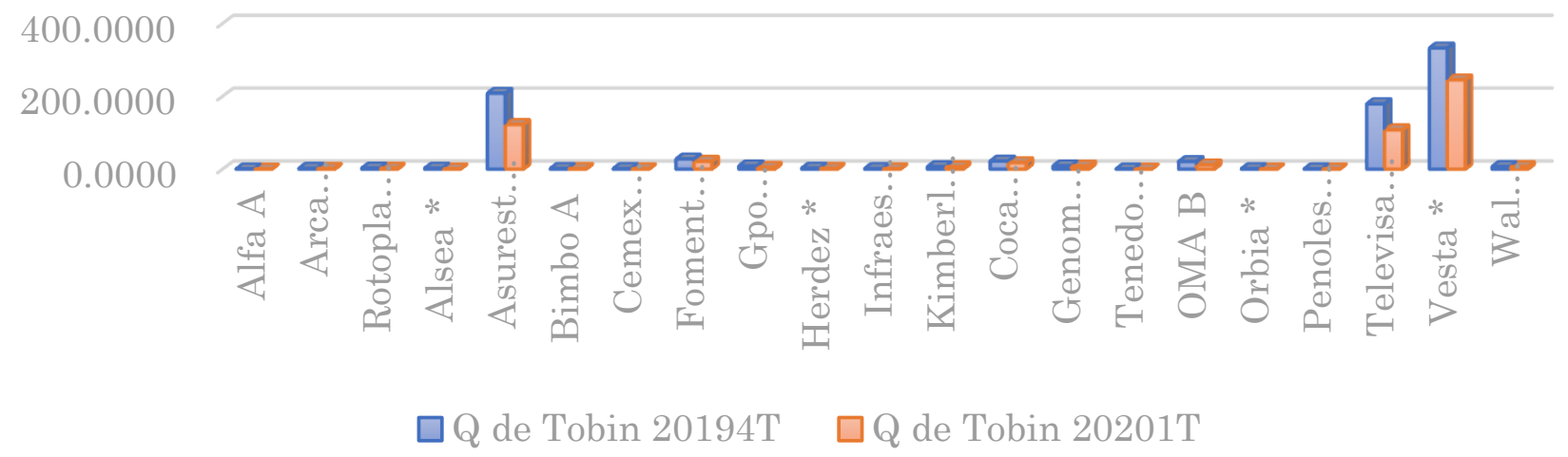

Figura 1. Comparativo de Q de Tobin.

Nota. Elaboración propia a partir de datos obtenidos de Economatica (2020).

En la Figura 2 se presentan las empresas seleccionados y el comportamiento de la $\mathrm{Z}$ de Altman, modelo que predice con anterioridad la quiebra de las empresas que conforman el IPC'S, con ello ayudan a las empresas e inversionistas a identificar si estas toman las decisiones acertadas para seguir operando, en este caso las siguientes empresas tienen una $\mathrm{Z}<1.81$ : Alfa A, Arca Continental, Rotoplas*, Alsea*, Asureste B, Bimbo A, Cemex CPO, Fomento Econ Mex UBD, Gpo Aeroport Pacif B, Herdez*, 
Infraes Energetica*, Kimberly Clark Mex A, KOFUBL Coca Cola Femsa UBL, Genomma Lab Intern B, Tenedora Nemak A, OMA B, Orbia*, Penoles Industrias*, Televisa Gpo CPO, Vesta, con esto se predice que las empresa mencionadas se están administrando de forma inadecuada financieramente, por lo que se corre el riesgo de entrar en quiebra.

\section{Comparativo del Valor Z Score 20194T y 20201T}

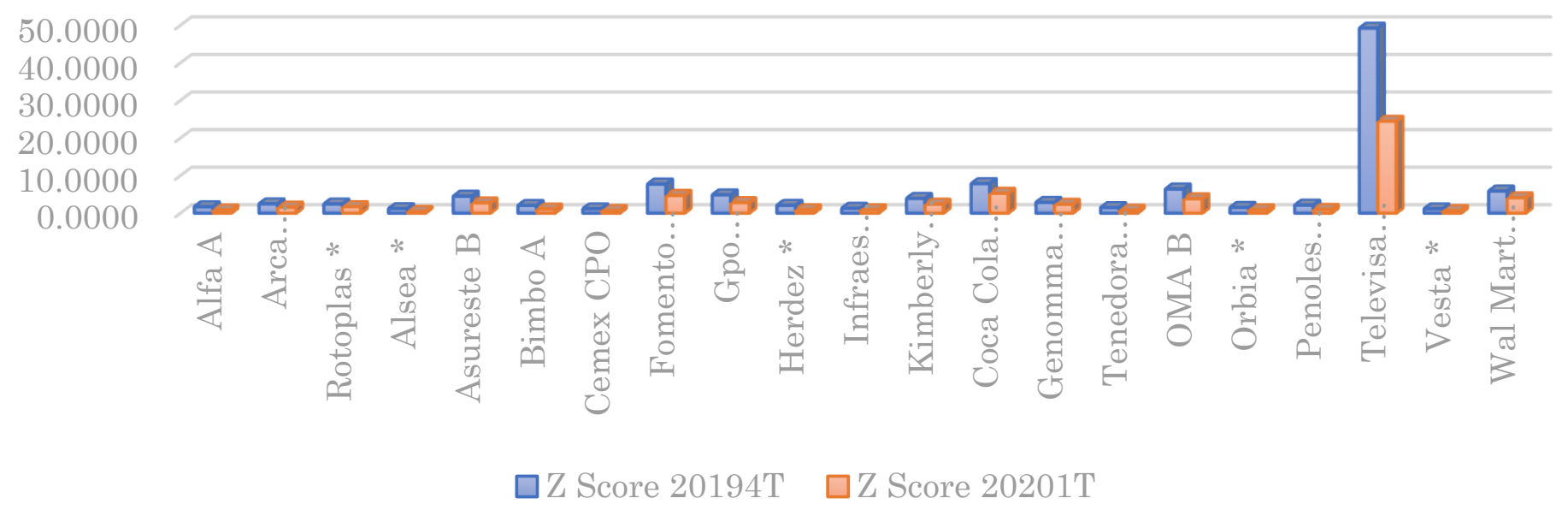

Figura 2. Comparativo de Z Score.

Nota. Elaboración propia con datos obtenidos de Economatica (2020).

\section{Resultados}

Citando a Morales, Aguilar y Monzón (2019), autores que consideran un grupo de 5 empresas socialmente responsables listadas en la Bolsa Mexicana de Valores, que se ubican en el sector productos de consumo frecuente, obtienen que para el periodo 2009-2017 4 de 5 empresas se mantienen en zona segura esto como resultado de su solidez financiera y para el caso de Bimbo se mantiene en promedio en la zona de precaución, con probabilidad alta de entrar en bancarrota, de acuerdo a la puntuación de la Z de Altman, ya que presenta a partir del 2014 al 2017 una Z por debajo de 2.99. Por otro lado, Walmex fue la única empresa en el periodo estudiado que mantuvo solidez financiera, concluyendo así que Wal-Mart México es la mejor empresa de las 5 analizadas.

De acuerdo con otra investigación desarrollada por Morales, Santiesteban y Monzón (2019), en el que hacen uso del IPC'S y del IPC de la Bolsa Mexicana de Valores para obtener los rendimientos a través del precio de la acción, consideran un grupo de 8 emisoras que forman parte del S\&P IPC-S de la BMV, con periodicidad de diciembre 2011 a agosto 2019 utilizando la rentabilidad logarítmica, con el objetivo de identificar si las empresas sustentables superan mes a mes al rendimiento presentado por el IPC de la BMV, encuentran periodos en los que el 54\% de las empresas superaban al índice en referencia. 
Estos autores al igual que la presente investigación desarrollan sus investigaciones utilizando empresas del índice de precios y cotizaciones sustentable que cotizan en la Bolsa Mexicana de Valores a través del valor de la acción.

En el caso de esta investigación uno de los elementos claves fue el comportamiento de las acciones de las empresas seleccionadas, pues esto nos indica el comportamiento que tienen en el mercado, a continuación, se brinda datos de las acciones (Tabla 4):

TABLA 4.

Precio de acción al cierre de cada trimestre

\begin{tabular}{llll}
\hline \multicolumn{1}{c}{ Emisora } & \multicolumn{1}{c}{$20191 \mathrm{~T}$} & $20194 \mathrm{~T}$ & \multicolumn{2}{l}{$20201 \mathrm{~T}$} \\
\hline Alfa SA A & 19.54 & 15.20 & 6.40 \\
Arca Continental, SAB de CV & 103.22 & 97.50 & 93.23 \\
Grupo Rotoplas S.A.B. de C.V. & 18.85 & 15.54 & 15.47 \\
Alsea SA & 40.67 & 49.83 & 15.18 \\
Grupo Aeroportuario del Sureste SAB de CV B & 159.69 & 224.67 & 127.39 \\
Grupo Bimbo S.A.B. & 39.58 & 33.94 & 33.99 \\
Cemex SA CPO & 8.84 & 7.08 & 4.93 \\
Fomento Económico Mexicano S.A.B. de C.V. & 177.78 & 178.67 & 143.22 \\
Grupo Aeroportuario del Pacifico, S.A.B. de C.V. & 159.69 & 224.67 & 127.39 \\
Grupo Herdez SA & 41.39 & 36.90 & 29.46 \\
Infraestructura Energética Nova S.A.B. de C.V. & 75.47 & 88.93 & 72.67 \\
Kimberly Clark de México S.A.B. de C.V. A & 31.21 & 37.07 & 35.92 \\
Coca-Cola Femsa SAB de CV UBL & 110.73 & 112.07 & 93.31 \\
Genomma Lab Internacional SA de CV & 13.97 & 18.71 & 18.87 \\
Nemak S.A.B. de C.V. & 10.25 & 7.76 & 3.98 \\
Grupo Aeroportuario del Centro Norte, S.A.B. de C.V. & 102.33 & 141.83 & 80.17 \\
ORBIA ADVANCE CORPORATION SAB de CV & 42.81 & 38.82 & 25.65 \\
Industrias Penoles & 233.96 & 198.21 & 157.88 \\
Grupo Televisa SAB CPO & 42.57 & 44.37 & 27.30 \\
Corporación Inmobiliaria Vesta, S.A.B. DE C.V. & 26.48 & 33.54 & 27.41 \\
Walmart de México SAB de CV & 50.06 & 53.88 & 55.48 \\
\hline & & & \\
\hline & & &
\end{tabular}

Fuente: Elaboración propia con datos obtenidos de Economatica (2020).

De las empresas seleccionadas para el estudio solo tres de ellas no presentan afectaciones en sus acciones: Kimberly Clark debido a que es una empresa dedicada a la fabricación, distribución y comercialización de Productos de limpieza, cuidado e higiene personal, con la presencia del COVID-19 el uso de material de limpieza aumento por las medidas de higiene que se adoptaron, en $20191 \mathrm{~T}$ el precio era de 31.21 y para el primer trimestre 2020 fue de 35.92. La segunda empresa es Genomma Lab, en donde se agrupa a empresas e instituciones que investigan, fabrican y comercializan productos farmacéuticos y dermocosméticos; con la presencia del COVID-19 se recurre a laboratorios, a la investigación farmacéutica, y es por eso por lo que la 
acción no se vio afectada, sino que presento un aumento en su acción de 13.97 en $20191 \mathrm{~T}$ a 18.87 para $20201 \mathrm{~T}$.

Y por último Walmart de México perteneciente al sector de productos de consumo frecuente en presencia del COVID-19 tuvo un aumento en la acción debido a la demanda de los consumidores, a pesar de las medidas precautorias para poder hacer compras esta empresa no cerro ya que cuenta con tienda en línea.

\section{Conclusiones}

Esta investigación estableció la siguiente hipótesis: Las empresas con criterios ESG que integran al S\&P/BMV Total México ESG Index tienen cada una de las acciones un precio infravalorado y no están en riesgo de quiebra, por lo cual hay oportunidad optima de inversión en ellas, por tanto una vez realizado los cálculos de la Q de Tobin y Z de Altman, se concluye con los resultados obtenidos de acuerdo con la Z de Altman de las empresas con criterios ESG que marca la investigación demuestran que han tenido un impacto considerable por los efectos ocasionados por la pandemia del COVID19, a pesar de que muchas de ellas cuentan con acciones inclusivas y sostenibles para el cuidado del medio ambiente y de la sociedad, que les ha llevado a que muchos de sus clientes compren sus productos y servicios, algunas de las presentes entidades como demuestran los cálculos entran en una zona de riesgo de quiebra, por lo cual tendrán que replantear las estrategias que tengan cada una de estas empresas, esto para evitar una quiebra de forma anticipada.

Si bien algunas empresas seleccionadas están en peligro (Alfa A, Tenedora Nemak A y Peñoles Industrias*), lo que indica que se tiene que desinvertir según la Q de Tobin, esto para ajustes progresivos para las empresas. Este modelo ha sido importante al momento de valorar y analizar de empresas de manera externa con el propósito de detectar oportunidades de inversión, internamente a la empresa le debe de interesar debido a que refleja las posibilidades de supervivencia de la empresa.

A partir de los resultados se dice que la $\mathrm{Z}$ score de Altman es una herramienta funcional que predice a partir de la situación financiera la quiebra de las empresas, y el buen funcionamiento de ellas, en este caso las empresas con mayor solidez y que son más atractivas para los inversionistas son cinco: Fomento Econ Mex UBD, Coca Cola Femsa UBL, OMA B, Televisa Gpo CPO y Wal Mart de México*, esto se debe al posicionamiento que tienen en la República Mexicana y a las estrategias que han tomado que se reflejan en los Estados de Resultados a pesar de la presencia del COVID-19.

\section{RECOMENDACIONES}

En tiempos de expansión de la empresa, se debe de tener consentimiento en lo que se está invirtiendo. Por lo tanto, las inversiones que se hagan deben de ser buenas y realmente planeadas, tener estrategias por parte de las empresas para que no se vean afectados sus estados financieros de forma drástica, especialmente en la parte de ventas y en la reducción de los costos como de deuda. Es por ello por lo que hay que tomar en cuenta las amenazas y oportunidades que se puedan presentar cuan- 
do se arrojen los resultados (Mata, 2020), esto para tomar decisiones adecuadas que ayuden a los inversionistas a generar estrategias para hacer crecer su patrimonio.

Al momento de valorar y analizar empresas, la $Q$ de Tobin es de relevancia ya que nos muestra la valoración de las empresas, es decir cómo la ve el mercado, si está sobrevaluada o subvaluada y con ella obtener resultados que nos brinden oportunidades de inversión ya que con ello se demuestra la estabilidad de las empresas, junto con este indicador tenemos la $\mathrm{Z}$ de Altman que nos brinda información de la salud financiera de la empresa y que por supuesto es parte relevante para los inversionistas.

Para concluir, a la hora de realizar inversiones el contar con conocimientos sobre los estados financieros, las acciones, conocer a las empresas en las que se quiera invertir y si estas se encuentran bien posicionada, si tiene crecimiento y es rentable, para con ello realizar inversiones de mejor forma.

\section{Agradecimientos y Reconocimientos}

El artículo "Pronóstico de quiebra y valuación de acciones en empresas sustentables de México" es el resultado del proyecto de investigación "Valuación Financiera y Empresarial de las Empresas Sustentables que cotizan en la Bolsa de Valores" y de la línea de Investigación que se lleva a cabo en la Facultad de Contaduría y Administración (FCA), UNAM.

\section{REFERENCIAS}

Altman, E. (1968). Financial ratios, discriminant analysis and the prediction of corporate bankruptcy. The journal of finance, 23(4), 589-609. https://doi. org/10.2307/2978933

Anjum, S. (2012). Business bankruptcy prediction models: A significant study of the Altman's Z-score model. Asian Journal of Management Researh, 3(1), 212-219. https://oi.org/10.2139/ssrn.2128475

BMV. (2020). Información de emisoras [Base de Datos]. México, D.F.: Grupo BMV. Disponible en https://www.bmv.com.mx/es/emisoras/informacion-de-emisoras

Chirinos, M., Fernández, L. y Sánchez, G. (2012). Responsabilidad empresarial o empresas socialmente responsables. Razón y Palabra, 16(2-81), 29-44. Disponible en https://ww.revistarazonypalabra.org/index.php/ryp/article/view/481

Economatica. (2020). Información de emisoras [Base de Datos]. Westbury: Economatica. Recuperado de https://economatica.com/

Langebaek, A. y Ortíz, J (2007). Q de Tobin y gobierno corporativo de las empresas listadas en bolsa. Borradores de Economía del Banco de la Republica de Colombia, 447, 1-38. Disponible en https:/www.banrep.gov.co/es/q-tobin-y-gobiernocorporativo-las-empresas-listadas-bolsa

Mata, J. (2020). La matriz FODA y su aplicación al trading e inversión en los mercados financieros. Yachana Revista Científica, 9(2), 27-38. Disponible en http:// revistas.ulvr.edu.ec/index.php/yachana/article/view/644 
Milei, J. G. (2011). Teoría de la inversión y mercados financieros: La "q" de Tobin y su uso para la valuación de empresas. Actualidad económica, 21(74), 7-17. Disponible en https://revistas.unc.edu.ar/index.php/acteconomica/article/view/3986

Morales, A., Aguilar, P. y Monzón, R. (2019). Salud financiera de las empresas socialmente responsables utilizando Z-Score de Altman. Yachana Revista Científica, 8(1), 41-59. Disponible en http://revistas.ulvr.edu.ec/index.php/yachana/ article/view/583

Morales, A., Santiesteban, J. y Monzón, R. (2019). Invertir en empresas con criterios ASG no significa renunciar a la rentabilidad. Un Espacio Para la Ciencia, 2(1), 103-130. Recuperado de https://07fb04e6-1a1e-490f-9f50-e41e85a00641.filesusr.com/ugd/fe0ad1_91c1c4be3ce24b4687c9dc290d79ba75.pdf

Paz, A., Sierra, L. y López, D. (2019). Responsabilidad Social: Dimensión sustentable hacia el ambiente de las empresas carboníferas en Colombia y Venezuela. Económicas CUC, 40(1), 47-60. https://doi.org/10.17981/econcuc.40.1.2019.03

Pérez, M., Chumaceiro, A. y Acosta, I. (2019). Sostenibilidad social en el sector agroindustrial. Un constructo para su desarrollo. Opción Revista de Ciencias Humanas y Sociales, 35(90), 100-149. Disponible en https://produccioncientificaluz. org/index.php/opcion/article/view/30460

Tobin, J. (1969). A General Equilibrium Approach to Monetary Theory. Journal of money, credit and banking, 1(1), 15-29. https://doi.org/10.2307/1991374

\section{BIODATA}

Arturo Morales Castro es Licenciado en Economía por la FE de la UNAM (México). Maestría en Finanzas, y Doctorado en Ciencias de la Administración por la FCyA-UNAM (México). Posdoctorado en la UAEMex y USAC (México). Líneas de investigación: Economía Financiera, Riesgos Financieros, Mercado de Derivados, Determinantes del Tipo de Cambio. ORCID: https://orcid.org/0000-0002-3159-5057

Sandra Avendaño Cruz es Licenciada en Economía por la FES Aragón (UNAM, México). Investigaciones basadas en temas relacionados con el tipo de cambio, índice sustentable, econometría, economía y finanzas. Estudiante de Maestría en Finanzas Corporativas por el Posgrado de la Facultad de Contaduría y Administración (UNAM, México). ORCID: https://orcid.org/0000-0002-2888-6136

José Miguel Mata Hernández es Licenciado en Negocios y Comercio Internacional por la Universidad Autónoma de Querétaro (México). Especialidad en Gestión de Riesgos Financieros y Empresariales EUDE (México). Actualmente se encuentra cursando la Maestría en Finanzas Bursátiles en el Posgrado de la Facultad de Contaduría y Administración de la Universidad Nacional Autónoma de México. Sus temas de investigación están enfocados a mercados financieros, el mercado de divisas, análisis y comportamiento de mercados, empresas de tecnología financiera. Es becario investigador en el Posgrado de la Facultad de Contaduría y Administración de la Universidad Nacional Autónoma de México. ORCID: https://orcid.org/00000003-0132-6881 